\title{
Acoustic Predictions in Industrial Spaces Using a Diffusion Model
}

\author{
Alexis Billon, ${ }^{1}$ Judicaël Picaut, ${ }^{2}$ Vincent Valeau, ${ }^{3}$ and Anas Sakout ${ }^{1}$ \\ ${ }^{1}$ LEPTIAB, University of La Rochelle, 17042 La Rochelle, France \\ ${ }^{2}$ Département IM UR EASE, Ifsttar Nantes, Route de Bouaye, CS 4, 44344 Bouguenais Cedex, France \\ ${ }^{3}$ Institut P' UPR 3346, LEA UMR 6609, Bâtiment K 40, Avenue du Recteur Pineau, 86022 Poitiers Cedex, France \\ Correspondence should be addressed to Alexis Billon, abillon@ulg.ac.be
}

Received 1 November 2011; Revised 16 January 2012; Accepted 19 January 2012

Academic Editor: C. N. Wang

Copyright () 2012 Alexis Billon et al. This is an open access article distributed under the Creative Commons Attribution License, which permits unrestricted use, distribution, and reproduction in any medium, provided the original work is properly cited.

Industrial spaces are known to be very noisy working environment. This noise exposure can be uncomfortable, tiring, or even harmful, at worst. Industrial spaces have several characteristics: they are often huge flat volumes fitted with many obstacles and sound sources. Moreover, they are usually surrounded by rooms where low noise levels are required. The existing prediction tools can seldom model all these phenomena accurately. In this paper, a prediction model based on a diffusion equation is presented. The successive developments carried out to deal with the various propagating phenomena met in industrial spaces are shown. For each phenomenon, numerical or experimental examples are given to highlight the validity of this model. It is also shown that its computation load is very little in comparison to ray-tracing-based methods. In addition, this model can be used as a reliable and flexible tool to study the physics of the coupling between rooms. Finally, an application to a virtual factory is presented.

\section{Introduction}

In industrial spaces, workers are often exposed to intense sound fields. So, for their security and comfort, reliable acoustic predictions must be obtained. Moreover, these industrial spaces are often surrounded by other rooms, such as offices, where low noise levels are required.

Industrial spaces present a number of acoustical characteristics. Firstly, they are huge volumes and so can hardly be treated using methods solving the propagation equation [1, 2 ]. Their height is often small compared to their length and width, giving birth to nondiffuse reverberated sound fields discarding methods based on the classical theory of reverberation [3]. They often contain many obstacles (machines, stockpiles, benches, etc.) which scatter and absorb the propagating sound: their acoustic behaviour of the room, when fitted, is very different from that of the same room, when empty.

To solve this particular characteristic, various prediction models have been developed including analytical models [4-10], empirical models [11], and simplified models [12]. Numerical models have also been developed, based on the ray-tracing concept [13]. However, the applications of these different models remain limited due to their assumptions concerning the obstacles or the enclosing room. The most flexible and reliable model was developed by Hodgson [14], extending the ray-tracing model of Ondet and Barbry [13] to take into account diffuse reflections at walls.

The ray-tracing method can treat coupling of rooms through aperture, but they seldom take into account coupling via transmission through common walls. Moreover, some important reflection paths can be missed in configurations composed of multiple connected spaces [15]. In industrial spaces, the number of sound sources can also be high leading to prohibitive computation times to obtain reliable results using the ray-tracing method.

Picaut et al. [16] have proposed a model, first derived by Ollendorff [17], to describe the local acoustic energy density in rooms with perfectly diffuse reflecting walls. By using a physical analogy with the diffusion of particles in a medium containing spherical scattering objects, as presented by Morse and Feshbach [18], they showed that the acoustic energy density may be the solution of a diffusion equation. This diffusion problem can be then analytically solved for one-dimensional configuration [19]. More recently, Valeau et al. [20] have generalized this model to three-dimensional enclosures and proposed a numerical implementation of this model for room-acoustic predictions. This model was 
successively extended to take into account the acoustic specificities met in industrial spaces. The diffusion model is derived from the more computationally demanding transport theory which is used to model the propagation of sound in street canyon [21] or in long rooms [22, 23].

In Section 2, the basic equation of the diffusion model is introduced. Section 3 presents the extension of this model to mixed specular/diffuse reflections. The atmospheric attenuation is taken into account in Section 4. The coupling through aperture and through partition walls is presented in Sections 5 and 6, respectively. Section 7 shows how scattering obstacles modify the diffusion model. The diffusion model can then be used as a tool to investigate the coupling between rooms, as presented in Section 8. Finally, an application to a virtual factory is presented in Section 9. Along this paper, numerical and experimental comparisons of the diffusion model are showed and computation times are indicated.

\section{Principle}

2.1. Diffusion Equation. For a room of volume $V$ and surface area $S$, the mean-free path of the room $\lambda$ can be evaluated by the simple analytical relation [24]

$$
\lambda=\frac{4 V}{S} .
$$

Following the physical analogy with the diffusion of particles in a scattering medium, the local acoustic energy density flux $\mathbf{J}(\mathbf{r}, t)$ can be approximated as the gradient of the acoustic energy density $w(\mathbf{r}, t)$ :

$$
\mathbf{J}(\mathbf{r}, t)=-D \operatorname{grad}(w(\mathbf{r}, t)),
$$

where the variables $\mathbf{r}$ and $t$ denote the position and time, respectively. $D$ is the diffusion coefficient, and its analytical expression is directly taken from the theory of diffusion for particles in a scattering medium:

$$
D=\frac{\lambda c}{3}
$$

where $c$ is the speed of sound. This term takes the room morphology into account through its mean-free path.

Let us consider the case of a room containing an acoustic omnidirectional point source located at position $\mathbf{r}_{s}$ and with an output acoustic power $P$. It can be shown from (2) that the acoustic energy density is the solution of the following diffusion equation [20]:

$$
\frac{\partial w(\mathbf{r}, t)}{\partial t}-D \nabla^{2} w(\mathbf{r}, t)=P(\mathbf{r}, t) \text { in } V
$$

In these equations, $\nabla^{2}$ is the Laplace operator and $V$ denotes the domain delimited by the room surfaces. In (4), the right-hand term is a source term which models the omnidirectional acoustic source in terms of power output and location [20]. This model assumes that the variations of energy density and energy flow remain small over one mean-free path $[16,18]$. This assumption implies that the receiving location must be sufficiently far the sound source in both time and space for the energy to scatter sufficiently, as pointed out by Navarro et al. [25].
2.2. Boundary Conditions. Equation (4) expresses the temporal and spatial evolutions of the acoustic energy density throughout the room and must be completed by boundary conditions taking into account the absorption acoustic energy at the room's surfaces. A common way of describing energy exchanges at a boundary is by the use of a mixed boundary condition, in the following form:

$$
\mathbf{J}(\mathbf{r}, t) \cdot \mathbf{n}=-D \frac{\partial w}{\partial \mathbf{n}}=h w(\mathbf{r}, t) \text { on } S,
$$

where $h$ is an exchange coefficient, similar to a convection coefficient in heat transfer. Firstly, this expression of the exchange coefficient was proposed [20]:

$$
h=\frac{c \alpha}{4}
$$

where $\alpha$ is the Sabine absorption coefficient. However, this boundary condition was limited to low absorption coefficients $(\alpha<0.2)$. To improve the obtained prediction for higher absorption coefficients, an exchange coefficient based on the Eyring absorption coefficient was later introduced $[26,27]$ :

$$
h=-\frac{c \ln (1-\alpha)}{4} .
$$

However, this expression becomes singular for $\alpha=1$. Using an analogy with light diffusion, Navarro et al. [25] obtained the following expression of the exchange coefficient:

$$
h=\frac{c \alpha}{2(2-\alpha)} .
$$

Equations (4) and (8) can also be obtained through the spherical harmonics' expansion of a transport equation [28] or a radiative transfer model [25]. This last derivation permits to show that the diffusion model can be considered as a natural extension of the geometrical acoustics assumptions [25]. Previously, Valeau et al. [20] demonstrated that the diffusion model can also be seen as an extension of the classical theory of reverberation to nondiffuse sound fields.

In the diffusion model, the boundary conditions state the frequency band under consideration by the modelling, as in other geometrical models. Changing the evaluated frequency band comes to change the values of the absorption coefficients. Otherwise, the absorption is described locally: each surface is described by its own absorption. This more accurate description is an advantage over the classical theory of reverberation theory which considers only a mean absorption coefficient per room.

2.3. Room Acoustics Criteria. The most critical room-acoustics criterion in industrial space is the sound pressure level. The diffusion equation models only the reverberated part of the sound field and the direct sound field must be added at a later stage. Thus, the sound pressure level (SPL) at a location r can be obtained with [20]:

$$
\operatorname{SPL}(\mathbf{r})=10 \times \log \left(\rho c\left(\frac{P}{4 \pi d^{2}}+c w(\mathbf{r})\right) \times \frac{1}{P_{\text {ref }}^{2}}\right),
$$



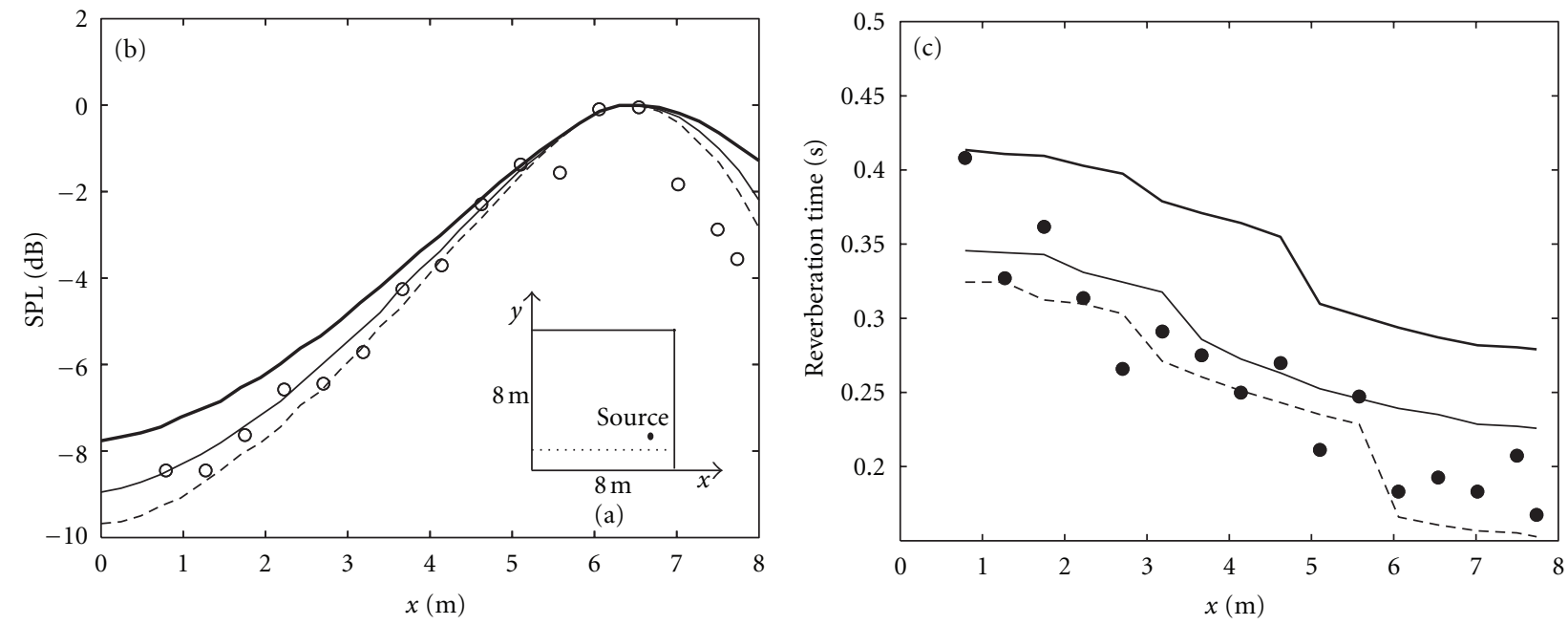

Figure 1: (a) Top view of the considered flat room. The black dot indicates the sound source, and the dotted line indicates the location of the measurements. (b) Evolution of the sound pressure level at the $1 \mathrm{kHz}$ octave band. (c) Evolution of the reverberation time at the $1 \mathrm{kHz}$ octave band [34]. (•) Experimental data, (-) diffusion model with (6) boundary condition, (- -) diffusion model with (7) boundary condition, and (-) diffusion model with (8) boundary condition.

where $d=\left|\mathbf{r}-\mathbf{r}_{s}\right|$ is the distance between the receiver and the source, $\rho$ the air density, and $P_{\text {ref }}=2 \times 10^{-5} \mathrm{~Pa}$.

Most of the room-acoustics criteria are related to the temporal behaviour of the impulse response [29]. The diffusion model assumes that the energy is sufficiently scattered. In mixing rooms, the sound field becomes diffuse after about three mean-free time (a mean-free time is equal to $\lambda / c$, the time needed to travel a mean-free path) [30]. So the sound field ought to be sufficiently scattered at shorter times for diffusion model to be valid. Valeau et al. [20] state that only one mean-free time is necessary, whereas Xiang et al. [31] propose that two mean-free times are needed. A systematic comparison with a ray-tracing software in terms several room-acoustics criteria concludes that the sound decays evaluated by the diffusion model become valid after two mean-free times [32]. So the criteria based on the early part of the sound decay, such as the early decay time EDT, the definition $D_{50}$, or the clarity $C_{80}$, cannot be reliably predicted. On the other hand, the reverberation time evaluated from the backward integrated sound decay $[31,33]$ can be accurately evaluated as it will be shown in the following through several examples.

2.4. Resolution of the Diffusion Problem. For one-dimension configurations, Picaut et al. [19] obtained analytical solutions for the diffusion problem. Valeau et al. [20] proposed a numerical implementation using the finite element method, permitting to simulate more realistic three-dimension configurations. Using the diffusion model, the acoustic problem can be reduced to a classical conduction-convection heat transfer problem for which finite element softwares are many and well validated. Also, using unstructured mesh, even the most complex geometry can be treated. Moreover, the mesh can be rather coarse, with grid size about the mean-free path of the considered geometry [20]. So most problems imply only several thousands of degree of freedom and can be rapidly solved. For sound pressure levels, the stationary form of (4) can be solved and results are obtained in a few seconds for a given frequency. The sound decays are obtained in a few tens of seconds, solving the time-dependent form of (4). Moreover, the obtained results (SPL or sound decays) are evaluated throughout the whole calculation domain implying that no recalculation is needed if the receiver is moved.

2.5. Application to a Flat Room. The diffusion model associated to the three boundary conditions introduced in (6), (7), and (8) is compared to experimental data both in terms of sound pressure level and in terms of reverberation time for a $8 \times 8 \times 1.2 \mathrm{~m}^{3}$ flat room (for more details, see [34]). The three models agree well with the experimental data in terms of SPL, with a maximum deviation of about $2 \mathrm{~dB}$ (Figure 1). A good agreement is also found in terms of reverberation times with the boundary conditions of (7) and (8). This example shows that reliable predictions of both sound levels and reverberation times can be obtained even for a configuration presenting a sound field far from being diffuse.

\section{Mixed Specular/Diffuse Reflections}

To obtain a diffusion equation, the reflections at walls were assumed to be perfectly diffuse. However, the reflections by walls are usually a combination of diffuse and specular reflections [35]. Valeau et al. [36] pointed out that increasing the value of the diffusion coefficient $D$ permits better predictions when specular reflections. By systematically comparing the results of the diffusion model with a cone-tracing software, Foy et al. [37] proposed a modified diffusion constant $D_{m}$ :

$$
D_{m}=\kappa D,
$$

where $D$ is the theoretical diffusion constant given by (3) and $\kappa$ is a function depending only on $s$, the scattering coefficient 


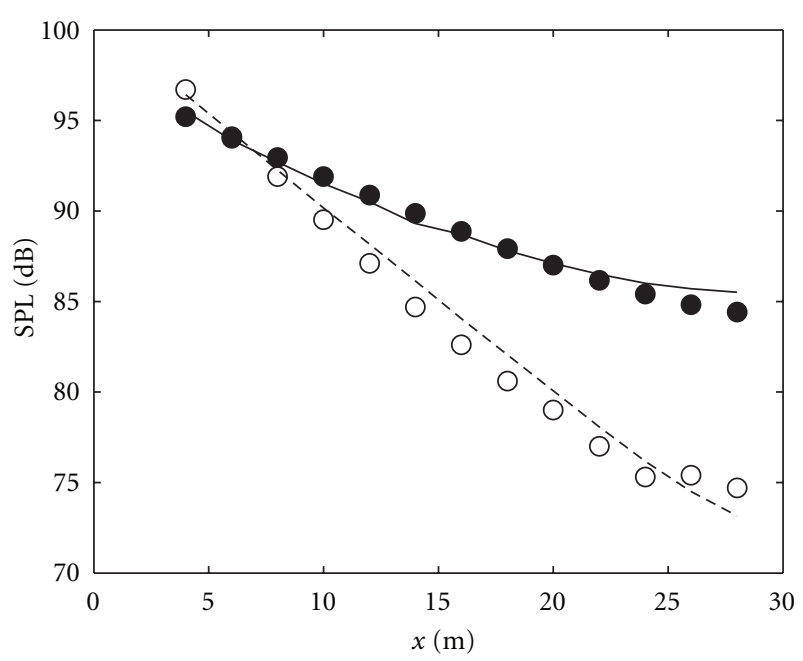

FIGURE 2: Evolution of the sound pressure (SPL) as a function of the receiver location in a centre axis of a long room of size $30 \times 2 \times 2 \mathrm{~m}^{3}$. $(\mathrm{o})$ and $(\bullet)$ diffusion model, (- $)$ and (-) cone-tracing software, for $s=0.2$ and $s=1$, respectively.

of the surfaces [38]. $\kappa$ is defined by the following empirical relation:

$$
\kappa=-2.238 \ln (s)+1.549 \quad \text { for } s>0
$$

It should be noted that, for $s=1$ (fully diffuse reflections), the modified diffusion constant is not equal to the theoretical one $(\kappa \neq 1)$ due to the empirical nature of the relation. Using this model, reliable results are obtained in terms of sound pressure levels whereas the reverberation times evaluated for $s<0.4$ are erroneous [33]. As an example, Figure 2 plots the SPL for a $30 \times 2 \times 2 \mathrm{~m}^{3}$ long room with $s=0.2$ and 1 showing the good agreement between the diffusion model and the cone-tracing software.

More recently, Visentin et al. [39] evaluated an estimated diffusion constant by comparing the acoustic energy intensity calculated by a particle-tracing and the diffusion model and obtained a spatially varying diffusion constant. In the vicinity of the source, the estimated diffusion constant is close to the one evaluated by (3) and it increases with the source distance [39]. This work introduces another more rigorous way to define a diffusion constant for specular reflecting surfaces.

\section{Atmospheric Attenuation}

The absorption of acoustical energy by the medium can have a significant impact on its propagation, particularly at high frequencies and for large rooms. An atmospheric attenuation term can be introduced in the diffusion model, leading to a modified expression of the diffusion constant [40]:

$$
D^{\prime}=D \times \frac{1}{1+m \lambda},
$$

where $m$ is the coefficient of atmospheric attenuation in $\mathrm{m}^{-1}$ and $D$ is the diffusion constant without atmospheric attenuation as defined in (3). However, the sound absorption

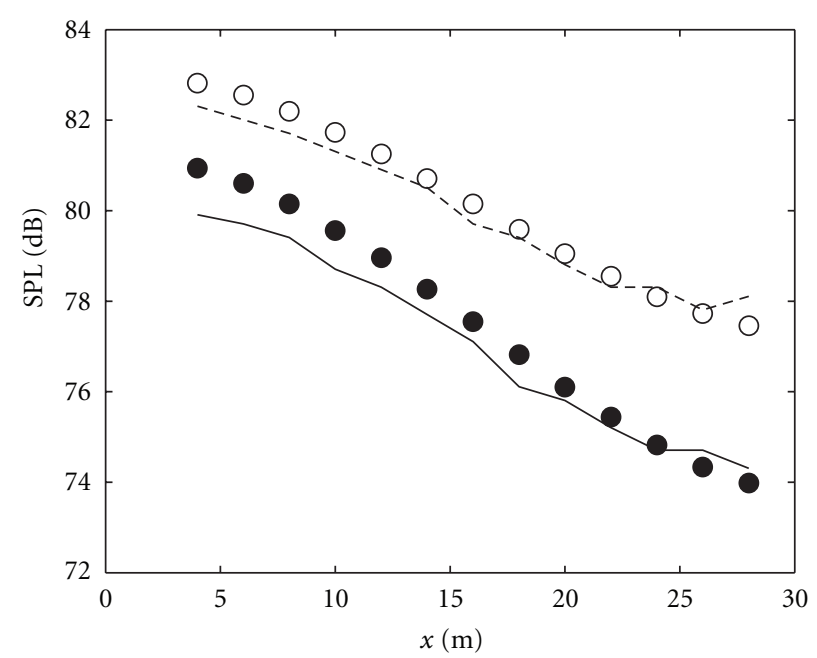

(a)

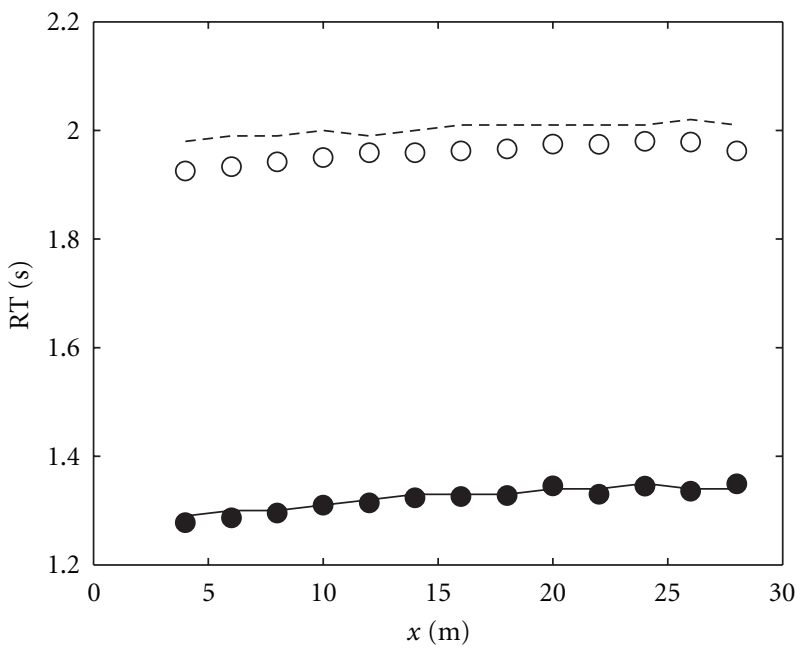

(b)

FIgure 3: (a) Sound pressure level and (b) reverberation time as a function of the receiver location in a flat room of size $(30 \times 30 \times 3)$ $\mathrm{m}^{3}$ [40]: (o) and $(\bullet)$ diffusion model, $(--)$ and $(-)$ cone-tracing software, without and with atmospheric attenuation, respectively.

being usually very small over a mean-free path (i.e., $m \ll 1$ ), one can consider thereafter that $D^{\prime} \approx D$. The diffusion equation for the energy density $w$ in the room, with a sound source term $P(\mathbf{r}, t)$, becomes [37]:

$$
\frac{\partial}{\partial t} w(\mathbf{r}, t)-D \nabla^{2} w(\mathbf{r}, t)+m c w(\mathbf{r}, t)=P(\mathbf{r}, t) \text { in } V .
$$

In this equation, the attenuation term has the typical form of an absorption term within the diffusion framework. Expanding a radiative transfer model, Navarro et al. [25] end up with the same expressions. Figure 3 shows that a good agreement is obtained between the diffusion model and a cone-tracing software for a $30 \times 30 \times 3 \mathrm{~m}^{3}$ flat room both in terms of SPL and RT [40]. In this example, the atmospheric attenuation is set to 0 and $0.01 \mathrm{~m}^{-1}$ corresponding to a $7.2 \mathrm{kHz}$ acoustic wave propagating in the air at $20^{\circ} \mathrm{C}$ and with $50 \%$ of relative humidity [38]. 


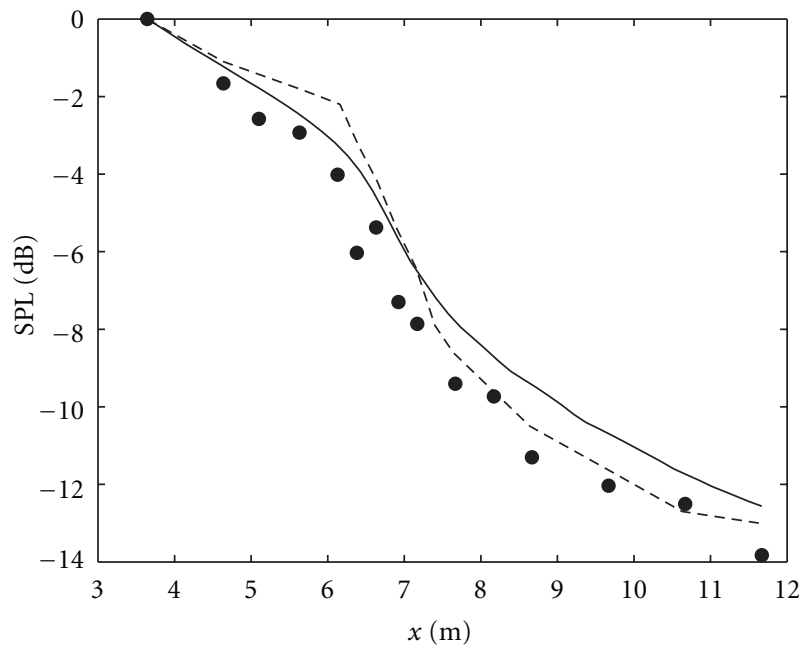

FIgURE 4: Evolution of the sound pressure level through the coupling aperture at the $1 \mathrm{kHz}$ octave band [42]: $(\bullet)$ experimental data, (-) diffusion model, and (- -) cone-tracing software.

\section{Coupling through an Aperture}

Coupled volumes systems, composed of two or more spaces that are connected through acoustically transparent openings (i.e., a coupling aperture), have attracted considerable attention in architectural acoustics. In concert halls, these configurations are of a particular interest, allowing to conciliate a high clarity with a strong reverberance thanks to doublesloped decays $[1,41]$. This configuration can also be found in various buildings or constructions such as industrial halls or office spaces.

For simulating the acoustics of two coupled rooms connected by an open aperture, a nonhomogeneous diffusion problem is to be solved where the computation domain $V$ defined by the two coupled rooms is decomposed into two subvolumes, $V_{1}$ and $V_{2}$ [42]. Moreover, it is assumed that the value of the mean-free path in each room is unaffected by the coupling. The following set of equations can then be written [42]:

$$
\begin{gathered}
\frac{\partial w(\mathbf{r}, t)}{\partial t}-D_{1} \nabla^{2} w(\mathbf{r}, t)=P(\mathbf{r}, t) \text { in } V_{1}, \\
\frac{\partial w(\mathbf{r}, t)}{\partial t}-D_{2} \nabla^{2} w(\mathbf{r}, t)=0 \text { in } V_{2},
\end{gathered}
$$

together with some boundary conditions. It should be pointed out that this method can be extended to an arbitrary number of coupled spaces without further assumptions or to an arbitrary of sound sources. Figure 4 plots results in the configuration of two coupled classrooms [42] showing the good agreement of the diffusion model with experimental data and a cone-tracing software in terms of SPL.

\section{Coupling through a Partition Wall}

Acoustical energy transferring from one room to another through partition walls can significantly deteriorate the building comfort. To evaluate this transfer, the standards are based on the theory of reverberation $[43,44]$ and their predictions are reliable as long as the assumptions of a diffuse sound field hold [3]. This energy transfer can be computed using the diffusion model. Firstly, the partition wall was assimilated as a surface source assuming a diffuse field in the room containing the source [20]. Later, the diffusion model was extended to solve the whole transmission problem and to consider nondiffuse sound fields on both sides of the partition wall [45]. Two diffusion equations must be considered, one for each enclosure:

$$
\begin{gathered}
-D_{1} \nabla^{2} w_{1}(\mathbf{r}, t)=P(\mathbf{r}, t) \text { in } V_{1}, \\
-D_{2} \nabla^{2} w_{2}(\mathbf{r}, t)=0 \text { in } V_{2},
\end{gathered}
$$

where $D_{1}$ and $D_{2}$ are the diffusion constants of the source and adjacent rooms, respectively. Note that only the stationary problem is considered here as the sound pressure level is the main criterion of interest in acoustic transmission problem.

At the coupling area, the energy exchanges between the rooms must be expressed as well as its absorption at its surface. Thus, the following boundary condition is obtained on the source room's side with a normal exterior vector $\mathbf{n}_{1}$ [45]:

$$
D_{1} \frac{\partial w_{1}}{\partial \mathbf{n}_{1}}+h_{1} w_{1}(\mathbf{r}, t)=\frac{\tau c}{4} w_{2}
$$

The left hand term is similar to (5) and depicts the loss of energy form the source room to the partition wall. The right hand term stands for the energy transferred from the adjacent room to the source room through the partition wall as a function of the transmission loss $R$ (or the transmission coefficient $\tau=10^{-R / 10}$ ). A similar boundary condition can be written on the adjacent room's side with a normal exterior vector $\mathbf{n}_{2}$ :

$$
D_{2} \frac{\partial w_{2}}{\partial \mathbf{n}_{2}}+h_{2} w(\mathbf{r}, t)=\frac{\tau c}{4} w_{1}
$$

The right hand term describes here the energy transferred from the source room. Similarly to the coupling through apertures, the principle can be extended to an arbitrary number of coupled rooms and sound sources. Otherwise, it can be shown that the energy balance obtained using the diffusion model can be reduced to the one obtained using the theory of reverberation, if a diffuse sound field is assumed in each room [45]. Figure 5 depicts the difference of sound level between two coupled classrooms [45]. A good agreement is between the measurement and the diffusion model.

\section{Diffusion by Scattering Objects}

As stated in the introduction, the industrial halls are seldom empty and contain many obstacles that scatter and absorb the sound energy during its propagation. This scattering objects can be modelled statistically and characterised by their density $n_{f}$ (i.e., the number of scattering objects per unit volume), their average scattering cross-section $Q_{f}$, and their absorption coefficient $\alpha_{f}[4,5]$. The scattering of sound by this obstacles can be then modelled by a diffusion process 


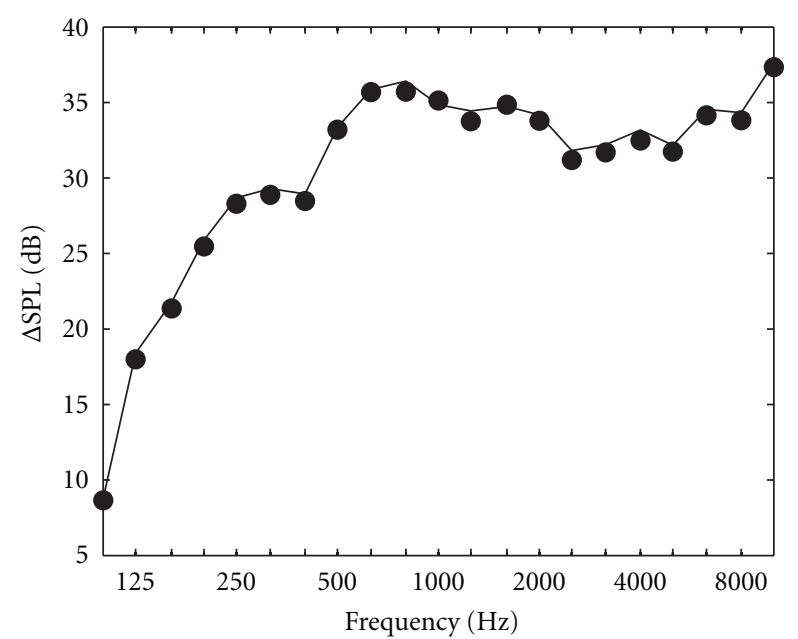

Figure 5: Sound pressure level difference by third octave band [45]: $(\bullet)$ experimental data and (-) diffusion model.

characterised by a mean-free path $\lambda_{f}=1 /\left(n_{f} Q_{f}\right)$ and a diffusion constant $D_{f}=\lambda_{f} c / 3$ [9]. The mean-free path of a sound particle travelling in a room characterised by a mean-free path $\lambda_{e}$ (for empty) and the scatterers previously described can be then written [32]:

$$
\lambda=\frac{\lambda_{e} \lambda_{f}}{\lambda_{e}+\lambda_{f}} .
$$

The diffusion equation for the acoustic energy density in a room containing scattering obstacles is then [36]:

$$
\frac{\partial w(\mathbf{r}, t)}{\partial t}-D t \nabla^{2} w(\mathbf{r}, t)+c \frac{\alpha_{f}}{\lambda_{f}} w(\mathbf{r}, t)=P(\mathbf{r}, t) \text { in } V
$$

with

$$
D_{t}=\frac{c\left(\lambda_{e} \lambda_{f}\right)}{3\left(\lambda_{e}+\lambda_{f}\right)}=\frac{D_{e} D_{f}}{D_{e}+D_{f}},
$$

where $D_{e}$ is the diffusion constant in the empty room a defined in (3). Equation (20) describes the combination of two diffusion processes, one due to the walls and the other due to the scattering objects. The additional term in (19) takes into account the absorption of sound energy by the scattering objects. Figure 6 shows that the obtained agreement of the diffusion model with the experimental data [13] is very good, similar to the one obtained with a raytracing software [36].

\section{Diffusion Model and Coupled Rooms}

Due to concert halls applications, systems of rooms coupled through apertures coupled volumes systems have attracted considerable attention. The diffusion model was shown to be a flexible and reliable tool to investigate the behaviour of these systems. It was firstly validated by comparison

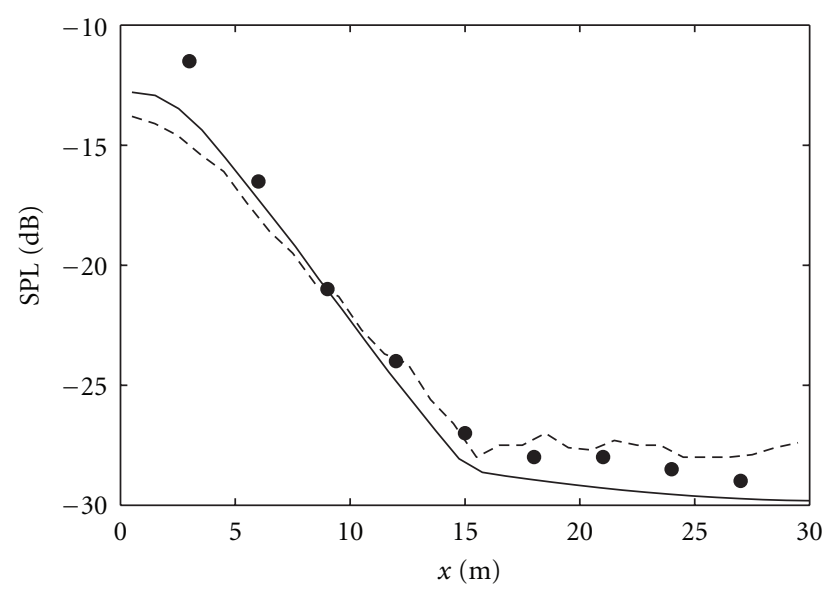

FIgure 6: Sound pressure level distribution [36]: $(\bullet)$ experimental data [13], (-) diffusion model, and (- -) ray-tracing software.

with statistical theory and experimental data [42]. It reliably models the gradual transition both in terms of sound pressure level (see Figure 5) and in terms of sound decays through the aperture. Jing and Xiang [46] used the diffusion model to study the energy flow from one room to another as a function of the time. It permits to observe that, for doublesloped decays, the energy flows back from the adjacent room to the source room. This reversal of the energy flow is associated with a dip of the energy-flow decay. These phenomena are not observed when the sound decays are not double-sloped. Moreover, Jing and Xiang [28] showed that the double-sloped decays can be accurately modelled by comparison with scale model measurements. It permits to link the energy flow reversal and the dip observed in its magnitude to the switching of slope in the measured sound decay. Pu et al. [47] observed that the double-sloped decays also occur in the adjacent room and is not restricted to the source room.

\section{Application to a Virtual Factory}

To exhibit the interest of the diffusion model to real-life applications, a configuration, similar to a small factory, is presented in Figure 7 [48]. This building is composed of a $20 \times 25 \times 10 \mathrm{~m}^{3}$ hall (A in Figure 7) connected through a $0.9 \times 2.1 \mathrm{~m}^{2}$ aperture to a $20 \times 2.5 \times 2.5 \mathrm{~m}^{3}$ corridor (B). The walls of the hall are in concrete $(\alpha=0.03)$, and the zone $\mathrm{A}^{\prime}\left(9 \times 7 \times 5 \mathrm{~m}^{3}\right)$ is fitted with scattering objects with absorption $\alpha_{f}=0.3$ and density $n_{f}=0.25$. Two $5.9 \times 3.5 \times 2.5 \mathrm{~m}^{3}$ offices $(\mathrm{D}$ and $\mathrm{E})$ are connected through $0.9 \times 2.1 \mathrm{~m}^{2}$ openings as well and a $8 \times 3.5 \times 2.5 \mathrm{~m}^{3}$ workshop (E) through a $2 \times 2.1 \mathrm{~m}^{2}$ opening. Four sound sources with different sound power level are located in the hall A: source 1 $(120 \mathrm{~dB})$ and sources 2,3 , and $4(100 \mathrm{~dB})$. Another $100 \mathrm{~dB}$ source is located in the workshop (5). The atmospheric sound attenuation is set to $0.005 \mathrm{~dB} / \mathrm{m}$.

In this study, two configurations of the corridor are compared. In the first configuration, the corridor is specularly reflecting $(s=0.2)$ and its absorption is homogeneous 


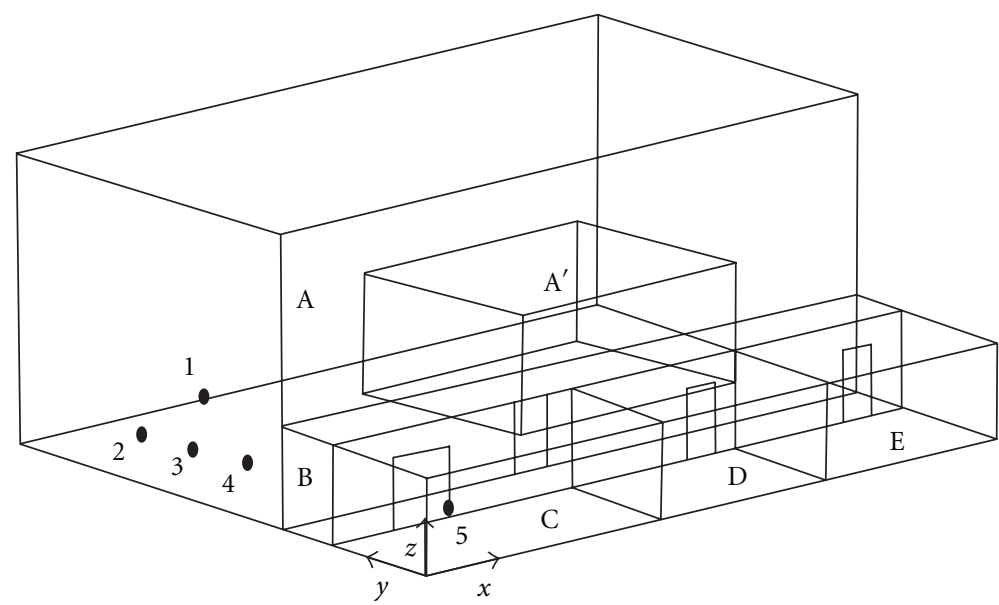

Figure 7: Sketch of the simulated building. A is an industrial hall where A' represents a volume fitted with scattering objects. B is a corridor, $\mathrm{C}$ a small workshop, $\mathrm{D}$ and $\mathrm{E}$ two offices. The numbers $1-5$ indicate the sound sources.

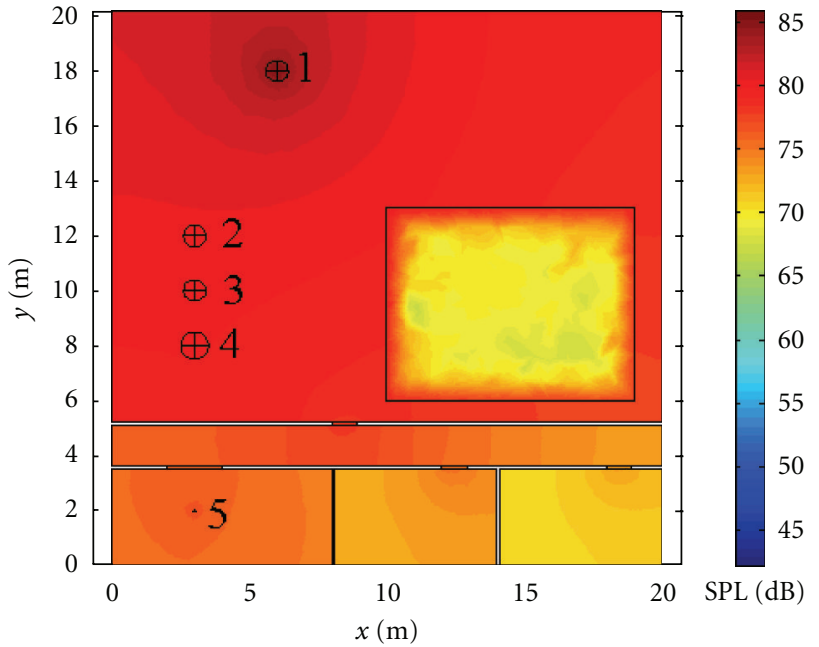

(a)

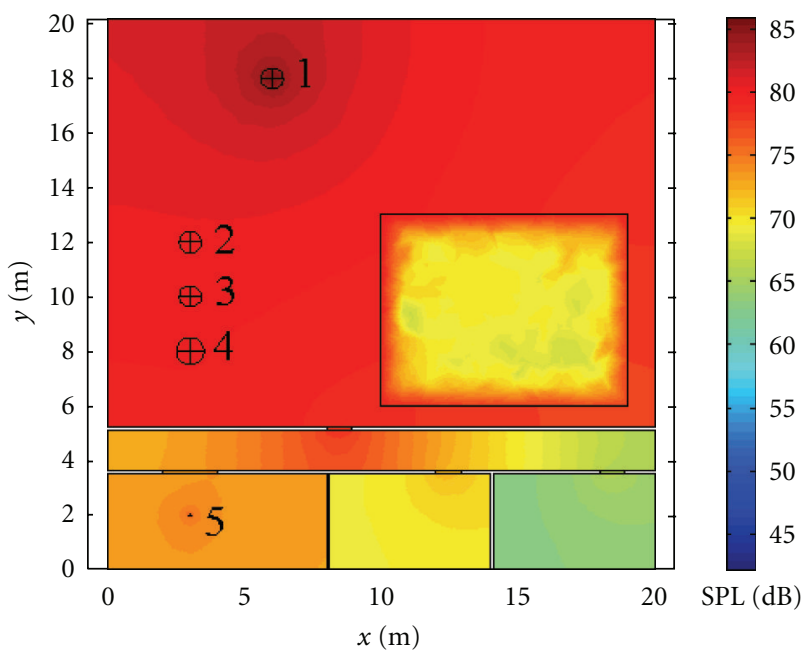

(b)

Figure 8: Color maps of the sound pressure level (SPL) of the reverberated sound field at $1.2 \mathrm{~m}$ : (a) nontreated configuration, (b) treated configuration.

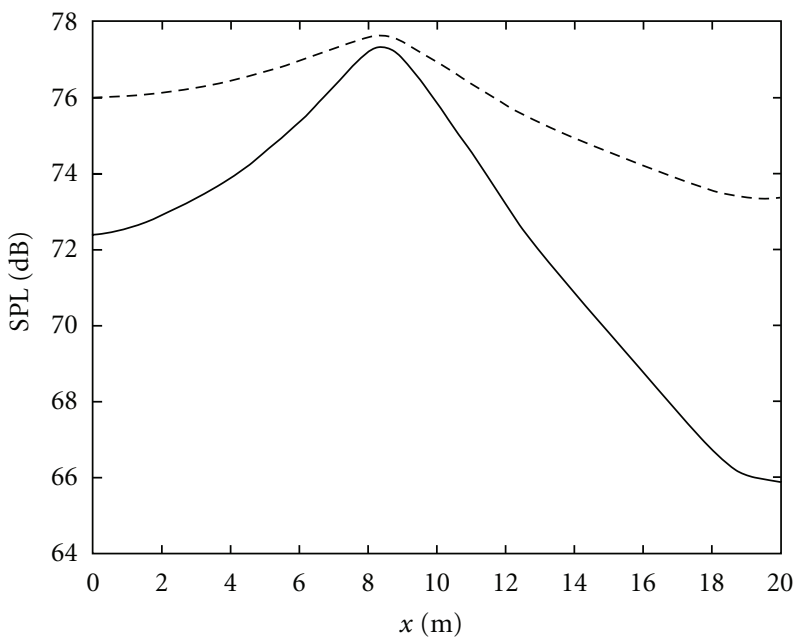

Figure 9: Sound pressure level (SPL) along the corridor at $1.2 \mathrm{~m}$ : (- -) nontreated corridor, (-) treated corridor.

$(\alpha=0.06)$. In the second configuration, the corridor's surfaces are diffuse $(s=1)$ and the ceiling is absorbent $(\alpha=0.6)$.

The computation time is around $30 \mathrm{~s}$ for the steady state sound levels. Due to the geometrical complexity of the problem, a simulation carried out using a ray-tracing software would need a huge number of rays (and so a very long computing time to obtain) in order to obtain physically consistent results.

Figure 8 presents color maps of sound pressure level of the reverberated sound field at $1.2 \mathrm{~m}$ high for both configurations. In particular, one can observe the decrease of SPL in the fitting zone, in comparison with the SPL in the hall, due to additional absorption from the scattering objects. The effect of the treatment is also clearly effective in the connected rooms. For example, the SPL decreases of about $5 \mathrm{~dB}$ in room E. Figure 9, plotting the SPL along the corridor at $1.2 \mathrm{~m}$ high, allows one to observe more closely the effect of treatment. The increase of absorption and scattering 
raises the sound attenuation of more than $8 \mathrm{~dB}$. The acoustics treatment in the corridor is also responsible for the decrease of the sound energy within the offices (D and E).

\section{Conclusions}

Industrial spaces often consist in huge, flat volumes fitted with a large number of diffracting objects and numerous sound sources. Moreover, they are often connected to other rooms (like offices) through common walls or apertures. These characteristics lead to complex acoustic problem to solve.

In this paper, a numerical model based on a diffusion equation was reviewed. It has been shown that the successive developments of this model succeed to solve most of the met difficulties of such acoustical spaces: nondiffuse sound field, atmospheric attenuation, coupling through apertures, transmission through partition walls, and sound absorption by scattering objects. This model can also be extended to an arbitrary number of sound sources or coupled spaces. Numerical and experimental comparisons were presented for each phenomenon exhibiting the reliability of the proposed model, both in terms of sound pressure levels and in terms of reverberation times. Moreover, the computation time has shown to remain very limited in regards to classical geometrical acoustics software. This model was thus used as a flexible and reliable tool to investigate the behaviour of the coupled-rooms, giving a new understanding of their behaviour. A real-life application to a virtual factory was presented, showing the capacity of the diffusion model in complex industrial spaces.

However, the limitations of the model were also pointed out. It cannot reliably model the early part of the sound decay and so cannot predict room-acoustics parameters such as definition, clarity, or early decay time. In spaces covered with specularly reflecting surfaces, the diffusion model permits to predict reliable sound pressure levels but is unable to obtain physically consistent reverberation times. The model also cannot predict the diffraction occurring around sound barriers. Those are several of the remaining challenges to be solved in the future.

\section{Acknowledgment}

The authors would like to thank the Agence de l'Environnement et de la Maîtrise de l'Énergie (ADEME) for providing financial support to this work.

\section{References}

[1] L. Cremer and H. H. Muller, Principles and Applications of Room Acoustics, vol. 1, Applied Science, London, UK, 1978.

[2] H. Kuttruff, Room Acoustics, Spon, London, UK, 4th edition, 1999.

[3] M. Hodgson, "When is diffuse-field theory applicable?" Applied Acoustics, vol. 49, no. 3, pp. 197-207, 1996.

[4] S. Jovivic, Anleitung zur Vorausbestimmung des Schallpegels in Betriebsgebäuden, Müller-BBM GmbH, München, Germany, 1979.
[5] K. H. Kuttruff, "Sound decay in reverberation chambers with diffusing elements," Journal of the Acoustical Society of America, vol. 69 , no. 6, pp. 1716-1723, 1981.

[6] E. A. Lindqvist, "Sound attenuation in large factory spaces," Acustica, vol. 50, no. 5, pp. 313-328, 1982.

[7] M. Hodgson, Physical and theoretical models as tools for the study of factory sound fields, Ph.D. dissertation, University of Southampton, 1983.

[8] N. Auletta, "Contribution à l'étude de la propagation du son dans les locaux industriels encombrés-lère partie (etude théorique)," Revue d'Acoustique, vol. 75, pp. 477-487, 1985.

[9] U. J. Kurze, "Scattering of sound in industrial spaces," Journal of Sound and Vibration, vol. 98, no. 3, pp. 349-364, 1985.

[10] P. Janeček, "A model for the sound energy distribution in work spaces based on the combination of direct and diffuse sound fields," Acustica, vol. 74, pp. 149-156, 1991.

[11] N. Heerema and M. Hodgson, "Empirical models for predicting noise levels, reverberation times and fitting densities in industrial workrooms," Applied Acoustics, vol. 57, no. 1, pp. 51-60, 1999.

[12] M. Hodgson, "Experimental evaluation of simplified models for predicting noise levels in industrial workrooms," Journal of the Acoustical Society of America, vol. 103, no. 4, pp. 19331939, 1998.

[13] A. M. Ondet and J. L. Barbry, "Modeling of sound propagation in fitted workshops using ray tracing," Journal of the Acoustical Society of America, vol. 85, no. 2, pp. 787-796, 1989.

[14] M. Hodgson, "On the accuracy of models for predicting sound propagation in fitted rooms," Journal of the Acoustical Society of America, vol. 88, no. 2, pp. 871-878, 1990.

[15] H. Lehnert, "Systematic errors of the ray-tracing algorithm," Applied Acoustics, vol. 38, no. 2-4, pp. 207-221, 1993.

[16] J. Picaut, L. Simon, and J. D. Polack, "A mathematical model of diffuse sound field based on a diffusion equation," Acustica, vol. 83, no. 4, pp. 614-621, 1997.

[17] F. Ollendorff, "Statistische raumakustik als diffusionsproblem," Acustica, vol. 21, no. 4, pp. 236-245, 1969.

[18] P. Morse and H. Feshbach, Methods of Theoretical Physics, McGraw-Hill, New York, NY, USA, 1953.

[19] J. Picaut, L. Simon, and J. D. Polack, "Sound field in long rooms with diffusely reflecting boundaries," Applied Acoustics, vol. 56, no. 4, pp. 217-240, 1999.

[20] V. Valeau, J. Picaut, and M. Hodgson, "On the use of a diffusion equation for room-acoustic prediction," Journal of the Acoustical Society of America, vol. 119, no. 3, pp. 1504-1513, 2006.

[21] T. Le Pollès, J. Picaut, M. Bérengier, and C. Bardos, "Sound field modeling in a street canyon with partially diffusely reflecting boundaries by the transport theory," Journal of the Acoustical Society of America, vol. 116, no. 5, pp. 2969-2983, 2004.

[22] Y. Jing, E. W. Larsen, and N. Xiang, "One-dimensional transport equation models for sound energy propagation in long spaces: theory," Journal of the Acoustical Society of America, vol. 127 , no. 4, pp. 2312-2322, 2010.

[23] Y. Jing and N. Xiang, "One-dimensional transport equation models for sound energy propagation in long spaces: simulations and experiments," Journal of the Acoustical Society of America, vol. 127, no. 4, pp. 2323-2331, 2010.

[24] W. B. Joyce, "Sabine's reverberation time and ergodic auditorium," Journal of the Acoustical Society of America, vol. 58, no. 3, pp. 643-655, 1975.

[25] J. Navarro, F. Jacobsen, J. Escolano, and J. Lopez, "A theoretical approach to room acoustic model based on a radiative transfer 
model," Acta Acustica United with Acustica, vol. 96, pp. 10781089, 2010.

[26] Y. Jing and N. Xiang, "A modified diffusion equation for room-acoustic predication (L)," Journal of the Acoustical Society of America, vol. 121, no. 6, pp. 3284-3287, 2007.

[27] A. Billon, J. Picaut, and A. Sakout, "Prediction of the reverberation time in high absorbent room using a modified-diffusion model," Applied Acoustics, vol. 69, no. 1, pp. 68-74, 2008.

[28] Y. Jing and N. Xiang, "Investigation on higher orders of spherical harmonics equations for efficient room-acoustics predictions," in Proceedings of the 19th International Congress on Acoustics (ICA '07), pp. 1-4, Madrid, Spain, 2007.

[29] "Acoustics-measurement of the reverberation time of rooms with reference to other acoustical parameters," International Organization for Standardization Document No. ISO 3382, 1997.

[30] A. Billon and J.-J. Embrechts, "Numerical evidence of mixing in rooms using the free path temporal distribution," Journal of the Acoustical Society of America, vol. 130, no. 3, pp. 13811389, 2011.

[31] N. Xiang, Y. Jing, and A. C. Bockman, "Investigation of acoustically coupled enclosures using a diffusion-equation model," Journal of the Acoustical Society of America, vol. 126, no. 3, pp. 1187-1198, 2009.

[32] J. Escolano, J. M. Navarro, and J. J. López, "On the limitation of a diffusion equation model for acoustic predictions of rooms with homogeneous dimensions," Journal of the Acoustical Society of America, vol. 128, no. 4, pp. 1586-1589, 2010.

[33] M. R. Schroeder, "New method of measuring reverberation time," Journal of the Acoustical Society of America, vol. 37, no. 1, pp. 409-412, 1965.

[34] Y. Jing and N. Xiang, "On boundary conditions for the diffusion equation in roomacoustic prediction: theory, simulations, and experiments," Journal of the Acoustical Society of America, vol. 123, no. 1, pp. 145-153, 2008.

[35] M. Hodgson, "Evidence of diffuse surface reflections in rooms," Journal of the Acoustical Society of America, vol. 89, no. 2, pp. 765-771, 1991.

[36] V. Valeau, M. Hodgson, and J. Picaut, "A diffusion-based analogy for the prediction of sound fields in fitted rooms," Acta Acustica United with Acustica, vol. 93, no. 1, pp. 94-105, 2007.

[37] C. Foy, V. Valeau, A. Billon, J. Picaut, and A. Sakout, "An empirical diffusion model for acoustic prediction in rooms with mixed diffuse and specular reflections," Acta Acustica United with Acustica, vol. 95, no. 1, pp. 97-105, 2009.

[38] T. J. Cox, B. I. L. Dalenback, P. D'Antonio et al., "A tutorial on scattering and diffusion coefficients for room acoustic surfaces," Acta Acustica United with Acustica, vol. 92, no. 1, pp. $1-15,2006$.

[39] C. Visentin, V. Valeau, N. Prodi, and J. Picaut, "A numerical investigation of the sound intensity field in rooms by using diffusion theory and particle tracing," in Proceedings of the 19th International Congress on Acoustics (ICA '10), pp. 1-6, Sydney, Australia, 2010.

[40] A. Billon, J. Picaut, C. Foy, V. Valeau, and A. Sakout, "Introducing atmospheric attenuation within a diffusion model for room-acoustic predictions (L)," Journal of the Acoustical Society of America, vol. 123, no. 6, pp. 4040-4043, 2008.

[41] J. E. Summers, R. R. Torres, and Y. Shimizu, "Statisticalacoustics models of energy decay in systems of coupled rooms and their relation to geometrical acoustics," Journal of the Acoustical Society of America, vol. 116, no. 2, pp. 958-969, 2004.
[42] A. Billon, V. Valeau, A. Sakout, and J. Picaut, "On the use of a diffusion model for acoustically coupled rooms," Journal of the Acoustical Society of America, vol. 120, no. 4, pp. 2043-2054, 2006.

[43] "Acoustics - measurement of sound insulation in buildings and of building elements - part 4: field measurements of airborne sound insulation between rooms," International Organization for Standardization Document No. ISO 140-4, 1998.

[44] "Building acoustics—estimation of acoustic performance of buildings from the performance of elements-part 1: airborne sound insulation between rooms," European Committee for Standardization Document No. EN-12354-1, 2000.

[45] A. Billon, C. Foy, J. Picaut, V. Valeau, and A. Sakout, "Modeling the sound transmission between rooms coupled through partition walls by using a diffusion model," Journal of the Acoustical Society of America, vol. 123, no. 6, pp. 4261-4271, 2008.

[46] Y. Jing and N. Xiang, "Visualizations of sound energy across coupled rooms using a diffusion equation model," Journal of the Acoustical Society of America, vol. 124, no. 6, pp. EL360EL365, 2009.

[47] H. Pu, X. Qiu, and J. Wang, "Different sound decay patterns and energy feedback in coupled volumes," Journal of the Acoustical Society of America, vol. 129, no. 4, pp. 1972-1980, 2011.

[48] J. Picaut, V. Valeau, A. Billon, and A. Sakout, "Sound field modeling in architectural acoustics using a diffusion equation," in Proceedings of the 20th International Conference on Noise, pp. 1-8, Honolulu, Hawaii, USA, 2006. 

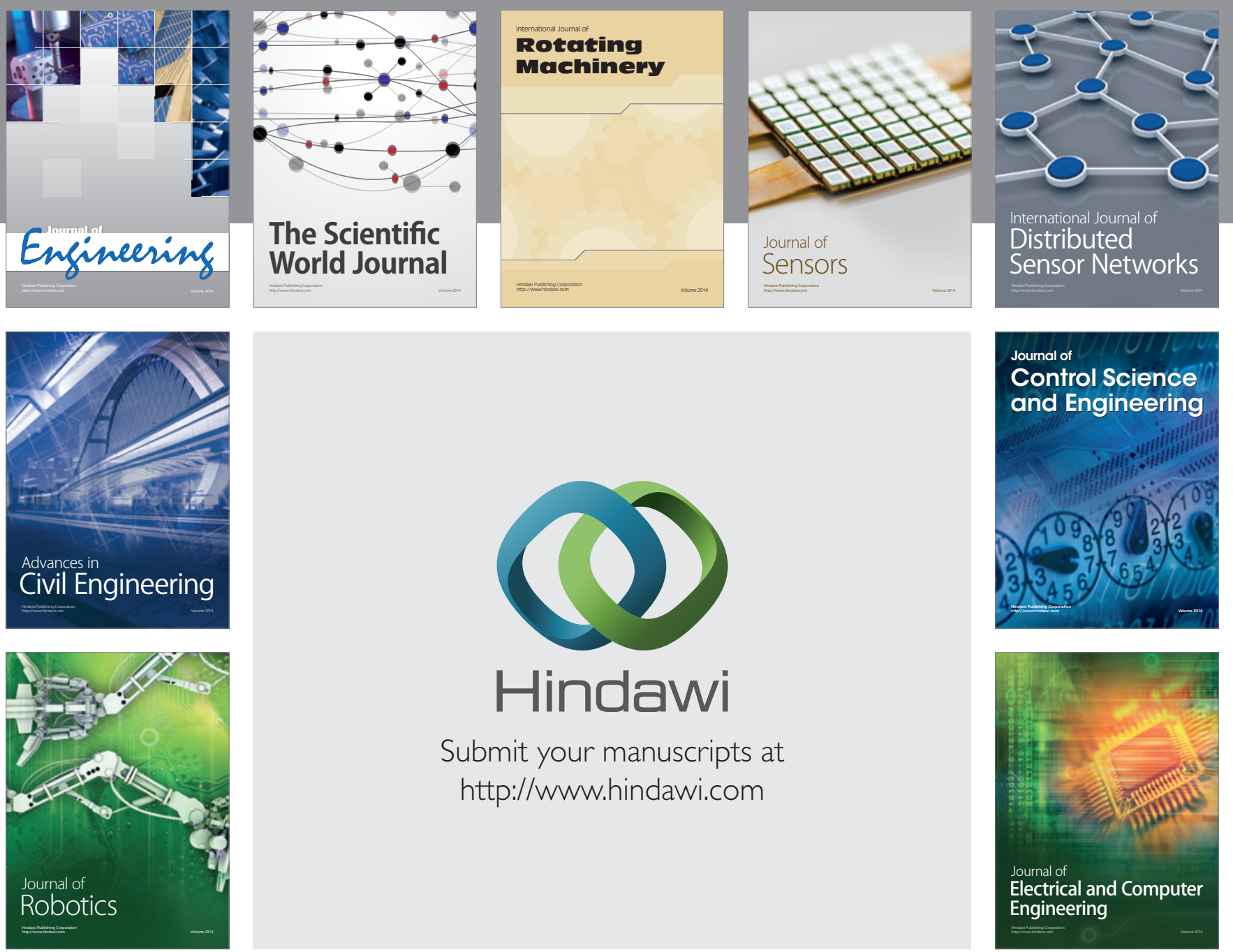

Submit your manuscripts at

http://www.hindawi.com
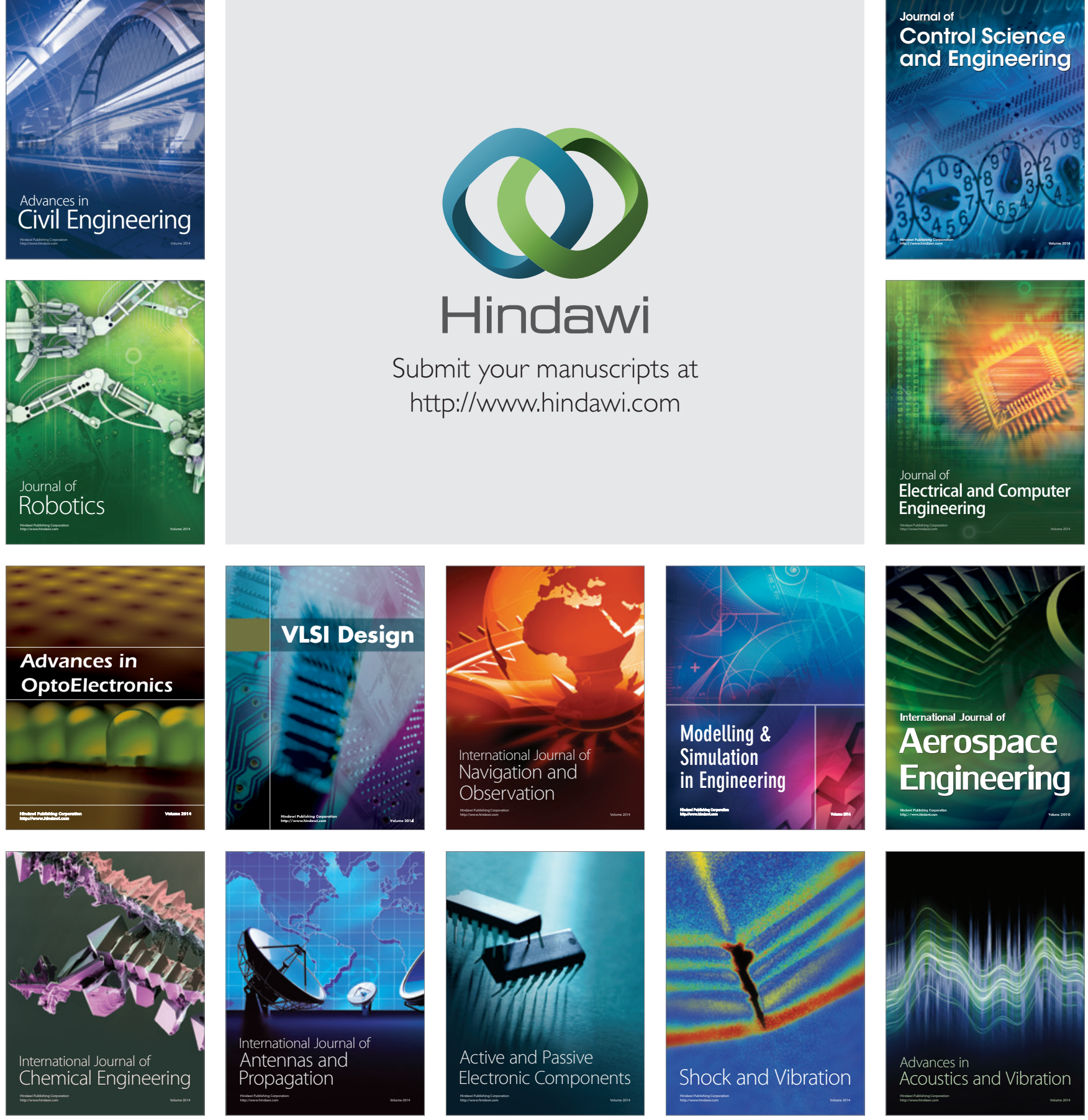